\title{
Non-destructive analysis of drug content in polymer coatings with Raman spectroscopy
}

\begin{abstract}
The analysis of device drug content typically is carried out by means of chromatographic methods such as high performance liquid chromatography (HPLC) or liquid chromatography-mass spectrometry (LCMS). These approved methods are particularly fast, cost-efficient and ubiquitous in chemical-analytical laboratories. However, these quantitative methods necessitate the drug being eluted, which represents a destructive process.
\end{abstract}

A novel alternative to these well-established methods $[1,2]$ is the Raman spectroscopy, which is fast and cost-efficient, as well [3]. Additionally, it offers the advantage of nondestructive analysis without the need for a special sample preparation. Within the current investigation we applied Raman spectroscopy for the qualitative and quantitative analysis of dexamethasone (DMS), a glucocorticoid, incorporated in a silicone matrix. The investigation was conducted in a rectangular area on the sample surface. The required number of measuring points (spectra) was determined. Calibration was performed with samples containing different amounts of DMS. The evaluation of Raman spectra is based on the analysis of the peak areas of the bands at $795 \mathrm{rel} . \mathrm{cm}^{-1}$ (silicone) and 1,663 rel. $\mathrm{cm}^{-1}$ (DMS). Remarkably, next to a precise overview of DMS distribution, an exact and reproducible quantification of incorporated DMS could be obtained.

Keywords: Raman spectroscopy, drug content, method optimizing

\footnotetext{
*Corresponding author: Thomas Reske: Institute for Implant Technology and Biomaterials e.V., Friedrich-Barnewitz-Str. 4, 18119 Rostock-Warnemünde, Germany, e-mail: thomas.reske@uni-rostock.de Katharina Wulf, Thomas Eickner, Niels Grabow: Institute for Biomedical Engineering, Rostock University Medical Center, Rostock-Warnemünde, Germany

Klaus-Peter Schmitz: : Institute for Biomedical Engineering, Rostock University Medical Center, Rostock-Warnemünde, Germany and Institute for Implant Technology and Biomaterials e.V., Rostock-Warnemünde, Germany

Stefan Siewert: Institute for Implant Technology and Biomaterials e.V., Rostock-Warnemünde, Germany
}

https://doi.org/10.1515/cdbme-2019-0118

\section{Introduction}

Raman spectroscopy represents a novel fast and cost-efficient alternative to well-established methods like HPLC and LCMS for the analysis of the drug content of samples. Furthermore, Raman spectroscopy offers the advantage of a non-destructive analysis that does not require a special sample preparation.

DMS and silicone are well evaluable by means of Raman spectroscopy. The investigation of the samples is not interfered by fluorescence. The analysed area depends on the specific sample geometry. The confocal Raman microscope used within the current study allows for $200 \mu \mathrm{m}$ measurements in the z-plane.

With regard to sample protection, laser output power has to be chosen carefully, typically ranging up to $50 \mathrm{~mW}$. To get a well-evaluable Raman spectrum, recorded within a short time, it must be well synchronized with the integration time.

\section{Materials and methods}

\subsection{Samples}

Silicone films with a thickness of about $1 \mathrm{~mm}$ and a DMS content of $5 \%, 10 \%, 15 \%$ and $20 \%$ were provided by the company MED-EL (MED-EL GmbH, Innsbruck, Austria), respectively.

\subsection{Overview image}

All measurements were performed using a confocal Raman microscope Alpha300R (WITec GmbH, Ulm). Overview images of samples with an area of 5,000 $\mu \mathrm{m}$ x 5,000 $\mu \mathrm{m}$ were recorded using a Zeiss EC "Epiplan-Neofluar" 10x objective (NA 0.25) (Carl Zeiss AG, Oberkochen, Germany). Therefore 256 single images were stitched. 


\subsection{Analysis via area scan measurement}

Raman measurements were performed within an area of $500 \mu \mathrm{m}$ x $500 \mu \mathrm{m}$ ("area scan") using $10 \times 10,25 \times 25$ and 50 x 50 measuring points, respectively. An integration time of $0.5 \mathrm{~s}$ per measuring point was applied. Laser output power of the Nd:YAG $532 \mathrm{~nm}$ laser ranges between 10 to $30 \mathrm{~mW}$. Measurements were also performed using the Zeiss EC "Epiplan-Neofluar" 10x objective.

\subsection{Raman data analysis}

Evaluation of the Raman spectra of every area scan was conducted in several steps within the software WITec Project 5.0:

- cosmic ray removal,

- baseline correction and

- calculation of an average spectrum.

Within the resulting average spectrum the area of the bands at $795 \mathrm{rel} . \mathrm{cm}^{-1}$ (silicone) and 1,663 rel. $\mathrm{cm}^{-1}$ (DMS) were determined via a Gauss fitting tool. Finally, the area ratio DMS / silicone was calculated.

\section{Results and discussion}

The Raman measurements were performed within an area of $500 \mu \mathrm{m} \times 500 \mu \mathrm{m}$ on the film samples. Figure 1 shows an exemplary overview image with such an investigated area of the $10 \%$ DMS sample.

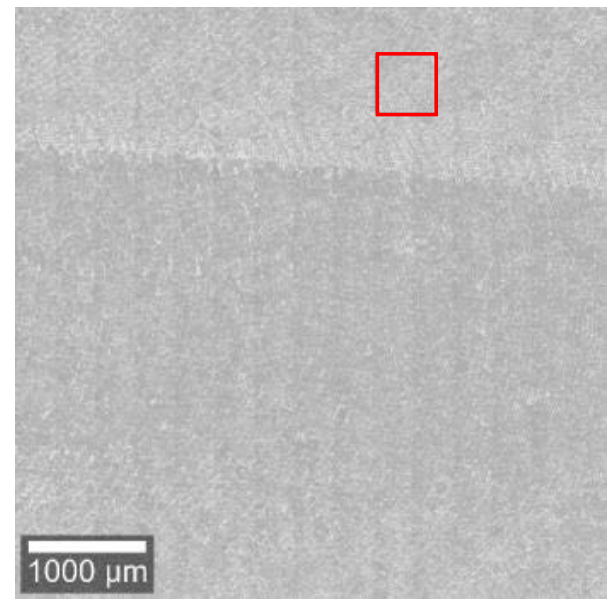

Figure 1: Exemplary overview image of a sample with $10 \%$ DMS content. The red box indicates the area where the Raman measurements were performed.

\subsection{Optimizing the laser output power}

For the Raman measurements an integration time of $0.5 \mathrm{~s}$ was defined before optimizing the laser output power. The sample thickness of about $1 \mathrm{~mm}$ is combined with good thermal conduction properties and allows the operator to use higher laser output power. Figure 2 shows the influence of the laser output power on the signal-to-noise ratio. Compared to 20 and $30 \mathrm{~mW}$, the ratio at $10 \mathrm{~mW}$ is high. Nevertheless the use of a high number of measuring points and its averaging decreases the signal-to-noise ratio. Within optimizing the laser output power 625 measuring points were used.

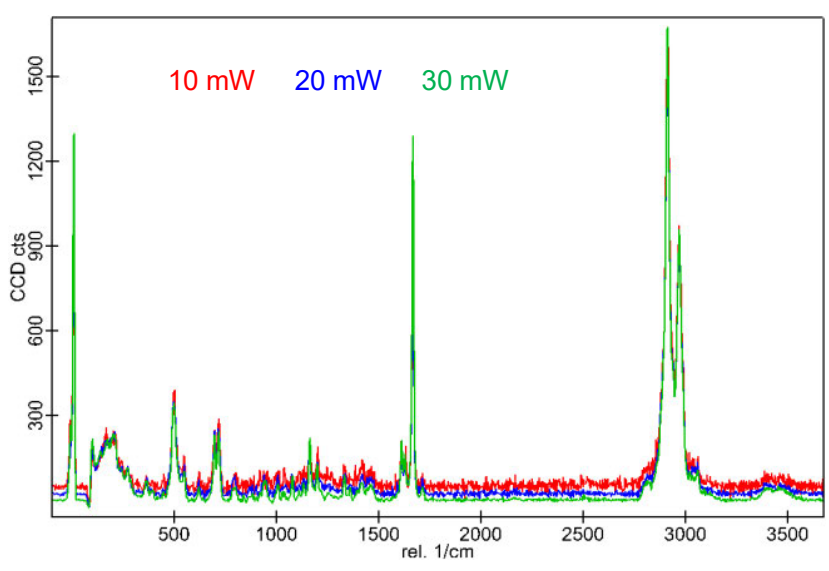

Figure 2: Raman spectra of randomly selected measuring points of sample with $20 \%$ DMS content at different laser output power.

Table 1 shows the results of the Raman measurements (detected using $25 \times 25$ measuring points at 10, 20 and $30 \mathrm{~mW}$ ).

Table 1: Areas and ratio of silicone and DMS bands for different laser output power and DMS contents.

\begin{tabular}{lcccc}
\hline $\begin{array}{l}\text { Laser output } \\
\text { power [mW] }\end{array}$ & $\begin{array}{c}\text { DMS } \\
\text { content } \\
\text { [\%] }\end{array}$ & $\begin{array}{c}\text { Area 795 } \\
\text { rel. } \mathbf{c m}^{-1} \\
\text { (silicone) }\end{array}$ & $\begin{array}{c}\text { Area } \\
\mathbf{1 , 6 6 3} \text { rel. } \\
\mathbf{c m}^{-1} \\
\text { (DMS) }\end{array}$ & $\begin{array}{c}\text { Ratio } \\
\text { DMS / } \\
\text { silicone }\end{array}$ \\
\hline 10 & 05 & 606 & 1,060 & 1.8 \\
10 & 10 & 597 & 1,830 & 3.1 \\
10 & 15 & 599 & 2,604 & 4.3 \\
10 & 20 & 576 & 3,574 & 6.2 \\
20 & 05 & 831 & 1,435 & 1.7 \\
20 & 10 & 1424 & 4,907 & 3.4 \\
20 & 15 & 736 & 3,502 & 4.8 \\
20 & 20 & 946 & 6,327 & 6.7 \\
30 & 05 & 2136 & 3,443 & 1.6 \\
30 & 10 & 1742 & 5,799 & 3.3 \\
30 & 15 & 1136 & 5,385 & 4.7 \\
30 & 20 & 1208 & 8,025 & 6.6 \\
\hline
\end{tabular}


Figure 3 shows that the averaging results in a linear correlation with correlation coefficients close to one.

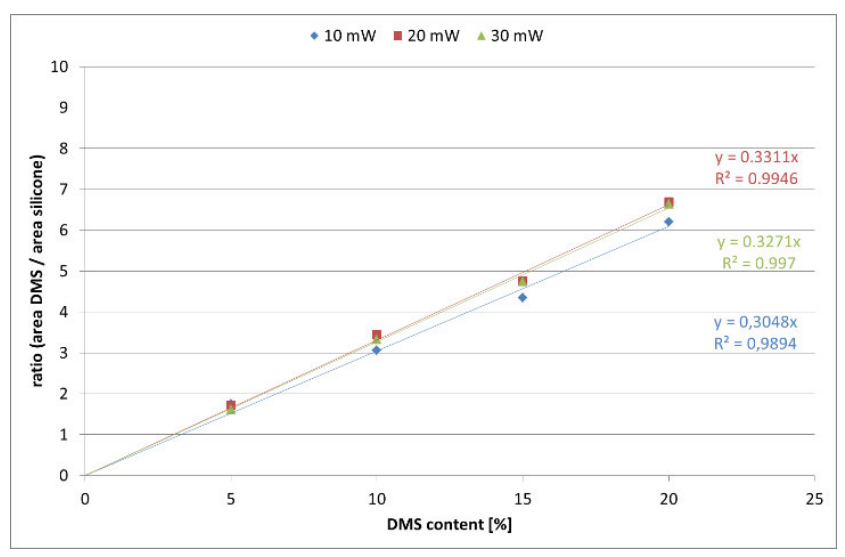

Figure 3: Dependency of the DMS / silicone ratio from the DMS content for different laser output power.

In the range of 10 to $30 \mathrm{~mW}$ the magnitude of laser output power does not influence the DMS / silicone ratio. Thus it was decided to perform further measurements with $10 \mathrm{~mW}$ while the integration time of $0.5 \mathrm{~s}$ per measuring point was maintained. Lower laser output power was not chosen because of lowering the signal-to-noise ratio.

\subsection{Optimizing the number of measuring points}

The number of measuring points ranged from $100(10 \times 10)$ to $2,500(50 \times 50)$. Hence the measurement time ranged from about 50 seconds (about 1 minute) to about 1,250 seconds (about 21 minutes). In Table 2 the resulting areas are compiled.

Table 2: Areas and ratio of silicone and DMS bands for different number of measuring points and DMS contents.

\begin{tabular}{lcccc}
\hline $\begin{array}{l}\text { Laser output } \\
\text { power [mW] }\end{array}$ & $\begin{array}{c}\text { DMS } \\
\text { content } \\
\text { [\%] }\end{array}$ & $\begin{array}{c}\text { Area 795 } \\
\text { rel. cm } \\
\text { (silicone) }\end{array}$ & $\begin{array}{c}\text { Area } \\
\mathbf{1 , 6 6 3} \text { rel. } \\
\text { cm }^{-1} \\
\text { (DMS) }\end{array}$ & $\begin{array}{c}\text { Ratio } \\
\text { DMS / } \\
\text { silicone }\end{array}$ \\
\hline 100 & 05 & 642 & 1,046 & 1.6 \\
100 & 10 & 559 & 1,830 & 3.3 \\
100 & 15 & 606 & 2,694 & 4.4 \\
100 & 20 & 544 & 3,247 & 6.0 \\
625 & 05 & 606 & 1,060 & 1.8 \\
625 & 10 & 597 & 1,830 & 3.1 \\
625 & 15 & 599 & 2,604 & 4.3 \\
625 & 20 & 576 & 3,574 & 6.2 \\
\hline
\end{tabular}

\begin{tabular}{lcccc}
\hline $\begin{array}{l}\text { Laser output } \\
\text { power [mW] }\end{array}$ & $\begin{array}{c}\text { DMS } \\
\text { content } \\
\text { [\%] }\end{array}$ & $\begin{array}{c}\text { Area 795 } \\
\text { rel. } \mathbf{c m}^{-1} \\
\text { (silicone) }\end{array}$ & $\begin{array}{c}\text { Area } \\
\mathbf{1 , 6 6 3} \text { rel. } \\
\mathbf{c m}^{-1} \\
\text { (DMS) }\end{array}$ & $\begin{array}{c}\text { Ratio } \\
\text { DMS / } \\
\text { silicone }\end{array}$ \\
\hline 2,500 & 05 & 574 & 1,008 & 1.8 \\
2,500 & 10 & 596 & 1,819 & 3.1 \\
2,500 & 15 & 524 & 2,380 & 4.5 \\
2,500 & 20 & 590 & 3,726 & 6.3 \\
\hline
\end{tabular}

DMS / silicone ratio is independent of the number of measuring points used, in the investigated range of 100 to 2,500. As Figure 4 shows, all parameters result in linear correlations with correlation coefficients close to one.

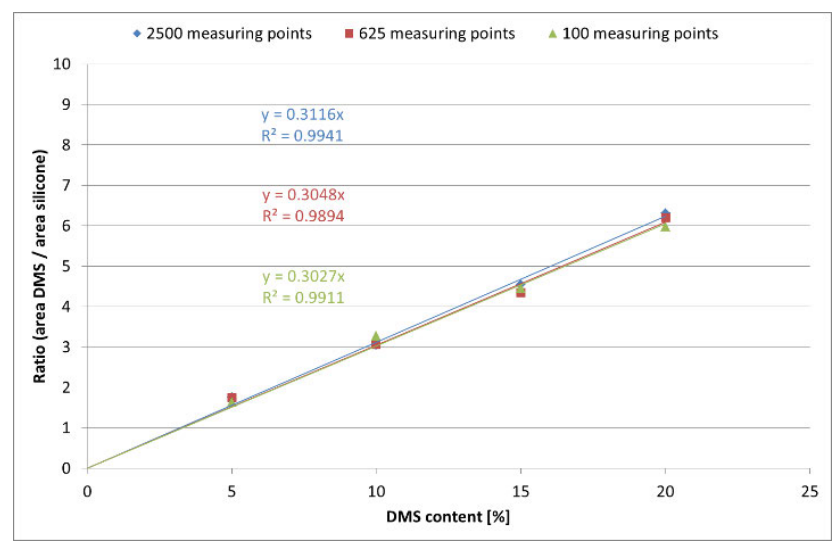

Figure 4: Dependency of the DMS / silicone ratio from the DMS content for different number of measuring points.

With a number of 100 measuring points the DMS content of a sample can be determined in the investigated range of 0 to $20 \%$ drug content. Using an integration time of $0.5 \mathrm{~s}$ such a measurement can be performed in less than one minute.

\section{Conclusion}

With a laser output power of $10 \mathrm{~mW}$ the samples can be investigated non-destructive. Even with a small number of 100 measuring points a linear correlation between the DMS content and the DMS / silicone ratio can be shown. Thus, analysis of one sample can be performed non-destructively in only one minute.

The good correlation coefficients indicate that the manufacturing of the samples was done with a high degree of homogeneity.

Future investigations can result in a further improvement of the method. With a decreased number of measuring points the measurement time can be shortened. With a smaller number of measuring points smaller areas can be 
investigated. Therefore, other sample geometries are also accessible to the Raman method.

Furthermore, the method may be applicable for the investigation of drug release. In that case it must be considered, that the drug is not distributed homogenously anymore.

Performing non-destructive Raman analysis permits the execution of additional (analytical) methods of the sample afterwards.

\section{Author Statement}

Research funding: Partial financial support by the European Regional Development Fund (ERDF) and by the Federal Ministry of Education and Research (BMBF) within RESPONSE "Partnership for Innovation in Implant
Technology" is gratefully acknowledged. Conflict of interest: Authors state no conflict of interest.

\section{References}

[1] Arthur KE, Wolff JC, Carrier DJ. Analysis of betamethasone, dexamethasone and related compounds by liquid chromategraphy/electrospray mass spectrometry. Rapid Commun Mass Sp 2004;18:678-685.

[2] Noben JP, Gielen B, Royackers E, Missotten M, Jacobs A, Raus J. A high-performance liquid chromatography/tandem mass spectrometric screening method for eight synthetic corticosteroids in bovine feces and the simultaneous differentiation between dexamethasone and betamethasone. Rapid Commun. Mass Sp 2002;16:1590-1594.

[3] Belu A, Mahoney C, Wormuth K. Chemical imaging of drug eluting coatings: Combining surface analysis and confocal Raman microscopy. J Control Release 2008; 126:111-121. 